\title{
Rate-Constrained Beamforming for Collaborating Hearing Aids
}

\author{
Olivier Roy \\ School of Computer and Communication Sciences \\ Ecole Polytechnique Fédérale de Lausanne (EPFL) \\ CH-1015 Lausanne, Switzerland \\ Email: olivier.roy@epfl.ch
}

\author{
Martin Vetterli ${ }^{1}$ \\ School of Computer and Communication Sciences \\ Ecole Polytechnique Fédérale de Lausanne (EPFL) \\ CH-1015 Lausanne, Switzerland \\ Email: martin.vetterli@epfl.ch
}

\begin{abstract}
Hearing aids are audio capture devices which aim at providing the hearing impaired with better audibility. Most of the state-of-the-art systems involve sensing devices that work independently. However, the availability of a wireless communication link between two hearing aids would allow to perform spatial beamforming such as to provide better rejection of interfering signals. In this paper, we identify and study the above scenario from an information-theoretic viewpoint. We explore the gain provided by collaborating hearing aids as a function of the communication rate. In particular, we derive a closed-form gainrate formula in the case where a sound source has to be extracted from ambient noise. A similar analysis is provided in the presence of an interfering point source and the corresponding optimal rate allocation is discussed.
\end{abstract}

\section{INTRODUCTION}

A digital hearing aid comprises three principal components: a set of microphones, a computing unit and a loudspeaker. Its goal is to process the acoustic signals acquired by the microphones such as to provide the loudspeaker with a signal that overcomes the user's hearing impairments. A possible task involves combining the incoming signals coherently in order to extract a sound source coming from a particular direction. This process is commonly referred to as beamforming and has been extensively studied over the last decades in the context of array processing [1]. The ability for an array of microphones to properly focus in one given direction depends on many parameters, such as the number of acquiring devices, the geometry of the array or the computing power available at the sensors. In particular, better resolution can be achieved by increasing the spatial extent of the array [2]. Limited by obvious design considerations, current hearing aids systems fail to provide good beamforming capabilities. This is mainly due to the fact that most equipments consist of two audio capture devices that work independently of each other. In this context, the availability of a wireless communication link between the two hearing aids would allow both devices to collaborate in order to achieve better speech intelligibility in noisy environments [3]

In this work, we look at the aforementioned problem from an information-theoretic perspective. We consider a hearing aid system where the two devices, each equipped with an

\footnotetext{
${ }^{1}$ Also with the Department of Electrical Engineering and Computer Sciences, University of California at Berkeley, Berkeley, CA 94720, USA.
}

omnidirectional microphone, are allowed to collaborate using a rate-constrained wireless link. In this sense, we study the impact of limited communication rates on the beamforming capability of a two-sensor array. Under the assumption of finite communication bandwidth, the beamforming paradigm studied in the array processing community [1], [2] simply corresponds to a multi-terminal scenario with a remote source and a particular correlation structure [4]. In this context, the setup considered in this paper is identified as being equivalent to a source coding problem with side information at the decoder where the source is merely observed in a remote fashion. This problem, referred to as remote, indirect or noisy Wyner-Ziv coding in the literature, has been addressed by various researchers [4], [5], [6] in the scalar case. Extension to vector sources was investigated in [7] in the context of high-rate transform coding. In this paper, we study both the approximation and compression perspectives for this matter and provide the results needed in order to cope with the problem at hand. Rate-constrained beamforming of jointly Gaussian stationary sources is then investigated for two cases of interest. In the first scenario, we wish to extract a point source from ambient noise. We derive a closed-form formula for the beamforming gain provided by our hearing aids setup as a function of the communication rate and the input signalto-noise ratio (SNR). In the second scenario, the presence of an additional interfering point source is considered and the corresponding gain-rate function is computed. Rate-allocation strategies are also discussed.

The outline of the paper is as follows: in Section II, we describe our hearing aids setup and identify the problem from an information-theoretic standpoint. Section III provides the necessary theoretical background. Gain-rate analysis along with optimal rate allocation strategies are presented for the two different scenarios of interest in Section IV. We finally offer some conclusions and future directions of research in Section V.

\section{THE HEARING AIDS PROBLEM}

The problem setup is depicted in Figure 1 for the two scenarios considered in this paper. It consists in a twosensor array where each acquiring device is equipped with an omnidirectional microphone, a processing unit with wireless 


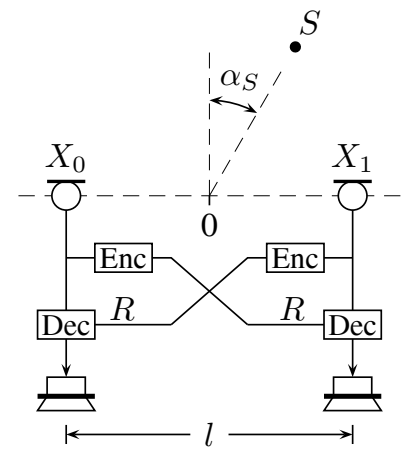

(a)

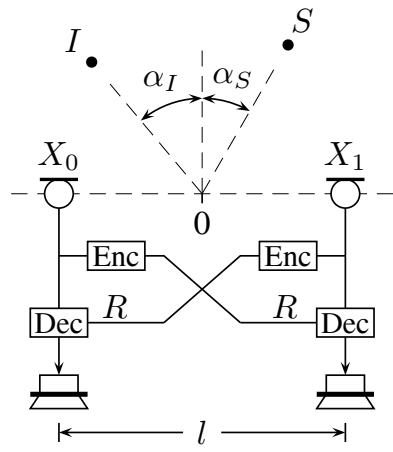

(b)
Fig. 1. Our hearing aids system. (a) Without any interferer. (b) With an interferer.

communications capabilities and a loudspeaker. The distance between the two hearing aids is $l$ and the origin of the axis is set in the middle of the array. For the scope of this paper, we will work under the far-field assumption ${ }^{1}$, i.e. that a point source impinging on the array can be accurately modelled as a plane wave with constant attenuation factor, set here to 1 . The point source $S$ of interest is an acoustic wave propagating at speed $c$ with a direction $\alpha_{S}$ known at both sensors. The signal acquired at each microphone is denoted $X_{0}$ and $X_{1}$, respectively. The involved sources are assumed to be zeromean continuous-time jointly Gaussian random processes with (real) bandlimited power spectral densities (PSD) $\Phi_{S}, \Phi_{X_{0}}$ and $\Phi_{X_{1}}$. The relationships between the different PSDs will be discussed in greater details in Section IV.

The goal of each hearing device is to beamform in the direction of $S$ in order to mitigate the effect of surrounding noise or interfering signals. To this end, each device receives a compressed version of its neighbor's acquired signal. We then aim at realizing a minimum mean-squared error (MMSE) beamformer. This choice is partly motivated by the fact that the optimality of this processor extends to various other criteria [1]. In this context, we wish to characterize the best achievable gain, at each hearing aid, that can be provided by the availability of a wireless communication link of rate $R$. The key is to realize that, under these assumptions, our setup simply corresponds to a remote Wyner-Ziv problem [5]. For a given rate $R$, we wish to encode $X_{1}$ such as to minimize the mean-squared error (MSE) between the source $S$ and its reconstruction $\hat{S}$, assuming the presence of some side information $X_{0}$ at the decoder. The corresponding distortionrate function is denoted $D(R)$. The gain achieved can thus be expressed as a function of $R$ as

$$
G(R)=\frac{D(0)}{D(R)} .
$$

The next section provides the results needed to compute

\footnotetext{
${ }^{1}$ The results of this paper equally apply to the near-field case but the farfield assumption greatly simplifies the derivations presented in the sequel.
}

the gain-rate function (1) for the scenarios considered in Section IV.

\section{Remote Source Coding With Side InFormation AT THE DECODER}

We consider the setup where an encoder observes a source $S$ remotely, i.e. by sensing $X_{1}$. It then sends a representation of $X_{1}$ to a decoder that computes a reconstruction $\hat{S}$ with the help of some side information $X_{0}$. In the spirit of [8], we look at this problem from two different viewpoints: approximation and compression.

In the approximation framework, we consider $S \in \mathbb{C}^{n}$, $X_{0} \in \mathbb{C}^{m_{0}}$ and $X_{1} \in \mathbb{C}^{m_{1}}$ to be zero-mean jointly Gaussian random vectors with covariance matrix $R_{S}, R_{X_{0}}$ and $R_{X_{1}}$, respectively. For simplicity, we will assume that $m_{0}, m_{1} \leq n$. The encoder provides a $k_{1}$-dimensional representation of $X_{1}$ by mean of a linear transform, i.e. it sends a transformed vector

$$
Y_{1}=K_{1} X_{1}
$$

where $K_{1} \in \mathbb{C}^{k_{1} \times m_{1}}$ with $k_{1} \leq m_{1}$. The decoder computes the reconstruction $\hat{S}$ based on $Y_{1}$ and $X_{0}$. The goal then is to design the transform $K_{1}$ such as to minimize the MSE between $S$ and $\hat{S}$. This problem was considered in [9] in the context of optimal linear fusion. In the next theorem, we provide a different solution from the one proposed in [9] which, we feel, gives more insights about the architecture of an optimal encoder. In line with the terminology adopted in [8], we call this transform the remote conditional Karhunen-Loève transform (rcKLT).

Theorem 1 (Remote Conditional KLT): The reKLT $K_{1} \in$ $\mathbb{C}^{k_{1} \times m_{1}}$ is given by

$$
K_{1}=\bar{K}_{1}^{*} R_{S X_{1}^{e}} R_{X_{1}^{e}}^{-1}
$$

where $\bar{K}_{1} \in \mathbb{C}^{n \times k_{1}}$ is the matrix whose columns are the $k_{1}$ eigenvectors of the matrix $R_{S}-R_{S \mid X_{1}^{e}}$ corresponding to the $k_{1}$ largest eigenvalues and where $X_{1}^{e}=X_{1}-\mathrm{E}\left[X_{1} \mid X_{0}\right]$. The resulting MMSE is computed as

$$
\mathrm{E}\left[\|S-\hat{S}\|^{2}\right]=\operatorname{tr}\left(R_{S \mid X_{0}, X_{1}}\right)+\sum_{i=1}^{m_{1}-k_{1}} \lambda_{i}
$$

where $\lambda_{i}$ denote the $m_{1}$ largest eigenvalues of $R_{S \mid X_{0}}-$ $R_{S \mid X_{0}, X_{1}}$ arranged in increasing order.

Proof: See Appendix.

In the above notation, $R_{X \mid Y}$ denotes the covariance matrix of the error vector $X-\mathrm{E}[X \mid Y]$. Theorem 1 shows that the optimal transform amounts to first compute the best estimate of $S$ (Wiener filtering) as if the error vector $X_{1}^{e}$ were available at the encoder and then simply apply a KLT on this estimate. In other words, it says that we should send the part of $S$ that can be predicted by $X_{1}$ but not by $X_{0}$. It is important to emphasize that the rcKLT is in general not unitary. Furthermore, it is seen in the proof of Theorem 1 that the availability of the side information at both the encoder and the decoder results in the same MMSE. In particular, the minimum distortion $\operatorname{tr}\left(R_{S \mid X_{0}, X_{1}}\right)$ 
corresponds to the part of $S$ that can be estimated neither by $X_{0}$ nor by $X_{1}$. Note that with appropriate assumptions, the rcKLT specializes to the transforms introduced in [8].

In the compression framework, the aforementioned problem can be recast as follows. Let $\{S[i]\}_{i=1}^{\infty},\left\{X_{0}[i]\right\}_{i=1}^{\infty}$ and $\left\{X_{1}[i]\right\}_{i=1}^{\infty}$ be discrete-time memoryless vector sources such that, for all $i, S[i] \in \mathbb{C}^{n}, X_{0}[i] \in \mathbb{C}^{m_{0}}$ and $X_{1}[i] \in \mathbb{C}^{m_{1}}$ are jointly Gaussian random vectors with mean zero and covariance matrix $R_{S}, R_{X_{0}}$ and $R_{X_{1}}$, respectively. Here again, $m_{0}, m_{1} \leq n$. The encoder senses $\left\{X_{1}[i]\right\}_{i=1}^{N}$ and outputs a bit stream that appears at a rate of $R$ bits per source vector. Based on the received data and the side information sequence $\left\{X_{0}[i]\right\}_{i=1}^{N}$, the decoder computes a reconstruction $\{\hat{S}[i]\}_{i=1}^{N}$ with averaged MSE $D_{N}(R)$ given by

$$
D_{N}(R)=\frac{1}{N} \sum_{i=1}^{N} \mathrm{E}\left[\|S[i]-\hat{S}[i]\|^{2}\right] .
$$

The goal then is to minimize the incurred distortion $D_{N}(R)$ for a given $R$ and characterize the optimal tradeoff $D(R)$ in the limit as $N \rightarrow \infty$. This problem was solved in the scalar case $\left(n=m_{0}=m_{1}=1\right)$ in [5]. Extension to vector sources was considered in [7] in the context of high rate transform coding. It was demonstrated that the optimal compression architecture can be split into a decorrelating transform followed by independent scalar Wyner-Ziv coding of each component. The authors of [7] showed that the optimal transform to apply in this context is specifically the rcKLT. In this sense, Theorem 1 also provides this transform with an operational meaning in the approximation framework. We compute in the next theorem the rate-distortion function for the problem at hand:

Theorem 2 (Remote Wyner-Ziv): The rate-distortion function for the remote Wyner-Ziv problem with vector sources is given in parametric form by

$$
\begin{aligned}
& R(\theta)=\sum_{i=1}^{m_{1}} \max \left\{0, \frac{1}{2} \log _{2} \frac{\lambda_{i}}{\theta}\right\} \\
& D(\theta)=\operatorname{tr}\left(R_{S \mid X_{0}, X_{1}}\right)+\sum_{i=1}^{m_{1}} \min \left\{\theta, \lambda_{i}\right\}
\end{aligned}
$$

where $\lambda_{i}$ are the $m_{1}$ largest eigenvalues of $R_{S \mid X_{0}}-R_{S \mid X_{0}, X_{1}}$ and $\theta \in\left(0, \max _{i} \lambda_{i}\right] . R(\theta)$ is expressed in units of bits per source vector and $D(\theta)$ in MSE per source vector.

Using arguments developed in [10], extension of Theorem 2 to continuous-time jointly Gaussian stationary processes with mean zero and bandlimited PSDs follows straightforwardly. The corresponding formulas are given here for future reference:

$$
\begin{aligned}
R(\theta)= & \frac{1}{4 \pi} \int_{-\infty}^{\infty} \max \left\{0, \log _{2} \frac{\Phi_{e}(\Omega)}{\theta}\right\} d \Omega \\
D(\theta)= & \frac{1}{2 \pi} \int_{-\infty}^{\infty} \Phi_{S \mid X_{0}, X_{1}}(\Omega) d \Omega \\
& +\frac{1}{2 \pi} \int_{-\infty}^{\infty} \min \left\{\theta, \Phi_{e}(\Omega)\right\} d \Omega
\end{aligned}
$$

where $\Phi_{e}=\Phi_{S \mid X_{0}}-\Phi_{S \mid X_{0}, X_{1}}$ and $\theta \in\left(0, \operatorname{ess}_{\sup _{\Omega}} \Phi_{e}(\Omega)\right]$. $R(\theta)$ is expressed in units of bits per second and $D(\theta)$ in MSE per second. In the above notation, $\Phi_{X \mid Y}$ denotes the PSD of the error process $X-\mathrm{E}[X \mid Y]$.

\section{RATE-CONSTRAINED BEAMFORMING}

The results derived in the previous section allow us to threat the hearing aids problem stated in Section II. The two cases of interest are presented in the next subsections.

\section{A. Ambient Noise}

We first aim at quantifying the gain provided by the availability of the wireless link when the point source $S$ is surrounded by ambient noise (see Figure 1(a)). This could be an appropriate model for a conversation in a noisy environment (e.g. in a crowded room). Under our far-field assumption, the signals $X_{0}$ and $X_{1}$ observed at the two microphones are expressed as

$$
\begin{aligned}
& X_{0}(t)=S\left(t-\tau_{0}\right)+N_{0}(t) \\
& X_{1}(t)=S\left(t-\tau_{1}\right)+N_{1}(t)
\end{aligned}
$$

where $N_{i}$ is a jointly Gaussian random process with mean zero and PSD $\Phi_{N_{i}}(i=0,1)$ and such that $N_{0}, N_{1}$ and $S$ are independent. The variable $\tau_{i}$ is the delay from the source $S$ to microphone $i$. We can easily show that

$$
\begin{aligned}
\Phi_{S \mid X_{0}} & =\frac{\Phi_{S} \Phi_{N_{0}}}{\Phi_{S}+\Phi_{N_{0}}} \\
\Phi_{X_{1}^{e}} & =\frac{\left(\Phi_{S}+\Phi_{N_{0}}\right)\left(\Phi_{S}+\Phi_{N_{1}}\right)-\Phi_{S}^{2}}{\Phi_{S}+\Phi_{N_{0}}} \\
\Phi_{S X_{1}^{e}} & =\frac{\Phi_{S} \Phi_{N_{0}}}{\Phi_{S}+\Phi_{N_{0}}} e^{-j \Omega \tau_{1}}
\end{aligned}
$$

where $X_{1}^{e}=X_{1}-\mathrm{E}\left[X_{1} \mid X_{0}\right]$. Using Lemma 1 given in Appendix, we can write

$$
\Phi_{e}=\frac{\Phi_{S}^{2} \Phi_{N_{0}}^{2}}{\left(\Phi_{S}+\Phi_{N_{0}}\right)\left(\Phi_{S} \Phi_{N_{0}}+\Phi_{S} \Phi_{N_{1}}+\Phi_{N_{0}} \Phi_{N_{1}}\right)}
$$

and

$$
\Phi_{S \mid X_{0}, X_{1}}=\frac{\Phi_{S} \Phi_{N_{0}}\left(\Phi_{S} \Phi_{N_{1}}+\Phi_{N_{0}} \Phi_{N_{1}}\right)}{\left(\Phi_{S}+\Phi_{N_{0}}\right)\left(\Phi_{S} \Phi_{N_{0}}+\Phi_{S} \Phi_{N_{1}}+\Phi_{N_{0}} \Phi_{N_{1}}\right)} .
$$

We will further assume that $S, N_{0}$ and $N_{1}$ have flat PSDs over the frequency band $\left[-\Omega_{0}, \Omega_{0}\right]$, i.e.

$$
\begin{aligned}
\Phi_{S}(\Omega) & =\sigma_{S}^{2} 1_{\left[-\Omega_{0}, \Omega_{0}\right]}(\Omega) \\
\Phi_{N_{i}}(\Omega) & =\sigma_{N}^{2} 1_{\left[-\Omega_{0}, \Omega_{0}\right]}(\Omega)
\end{aligned}
$$

for $i=0,1$. In this case, the distortion-rate function corresponding to Equations (8) and (9) can be computed in closedform as

$$
D(R)=\frac{\Omega_{0}}{\pi} \frac{\sigma_{S}^{2} \sigma_{N}^{2}}{2 \sigma_{S}^{2}+\sigma_{N}^{2}}\left(\frac{\sigma_{S}^{2}}{\sigma_{S}^{2}+\sigma_{N}^{2}} 2^{-2 \pi R / \Omega_{0}}+1\right)
$$

for $R \geq 0$. The gain-rate function follows by evaluating (1) using (19). We obtain

$$
G(R)=\frac{2 \sigma_{S}^{2}+\sigma_{N}^{2}}{\sigma_{S}^{2}+\sigma_{N}^{2}}\left(\frac{\sigma_{S}^{2}}{\sigma_{S}^{2}+\sigma_{N}^{2}} 2^{-2 \pi R / \Omega_{0}}+1\right)^{-1}
$$




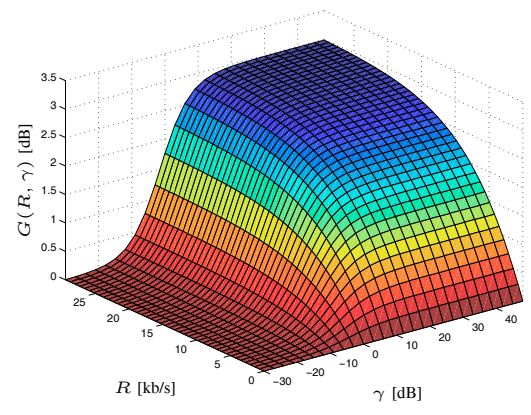

Fig. 2. Gain provided by the wireless communication link as a function of the communication rate $R$ and the input $\operatorname{SNR} \gamma$.

or as a function of the input SNR $\gamma=\sigma_{S}^{2} / \sigma_{N}^{2}$,

$$
G(R, \gamma)=\frac{2 \gamma+1}{\gamma+1}\left(\frac{\gamma}{\gamma+1} 2^{-2 \pi R / \Omega_{0}}+1\right)^{-1}
$$

The gain provided by allowing collaboration between the hearing aids is plotted in Figure 2 as a function of the communication rate and the input SNR. As $R \rightarrow \infty$, the gain remains bounded and corresponds to that of a two-sensor array with no rate constraint. At high SNR, this gain approaches $10 \log _{10}(K)[\mathrm{dB}]$ where $K=2$ is the number of sensing devices. We also observe that, in this scenario, the result depends neither on the actual position of the source nor on the geometrical properties of our hearing aids setup. This is due to the far-field assumption and the fact that the noise is uncorrelated across sensors. Note that a similar analysis can be carried in the case the involved PSDs are not flat. However, the corresponding gain-rate function must be generally left in a parametric form.

\section{B. Ambient Noise and Interfering Source}

In the presence of an additional interfering point source, the beamforming capability provided by collaborating hearing aids becomes crucial since it allows to separate signals with different directions of arrival. We assume the interfering signal $I$ to be an acoustic wave propagating at speed $c$ with a direction $\alpha_{I}$ known at both sensors (see Figure 1(b)). We model it as a zero-mean continuous-time jointly Gaussian random process with (real) bandlimited PSD $\Phi_{I}$. In this case, the signals observed at the microphones can be written as

$$
\begin{aligned}
& X_{0}(t)=S\left(t-\tau_{0}\right)+I\left(t-\tilde{\tau}_{0}\right)+N_{0}(t) \\
& X_{1}(t)=S\left(t-\tau_{1}\right)+I\left(t-\tilde{\tau}_{1}\right)+N_{1}(t)
\end{aligned}
$$

where $I$ is independent of $S$ and $N_{i}(i=0,1)$ and whose delay with respect to microphone $i$ is denoted $\tilde{\tau}_{i}$. Similarly to Section IV-A, $\Phi_{e}$ can be computed as

$$
\begin{gathered}
\Phi_{e}=\frac{\Phi_{S}^{2}}{\Phi_{S}+\Phi_{I}+\Phi_{N_{0}}} \\
\frac{2 \Phi_{I}\left(\Phi_{I}+\Phi_{N_{0}}\right) \Delta(\Omega)+\Phi_{N_{0}}^{2}}{2 \Phi_{S} \Phi_{I} \Delta(\Omega)+\left(\Phi_{N_{0}}+\Phi_{N_{1}}\right)\left(\Phi_{S}+\Phi_{I}\right)+\Phi_{N_{0}} \Phi_{N_{1}}}
\end{gathered}
$$

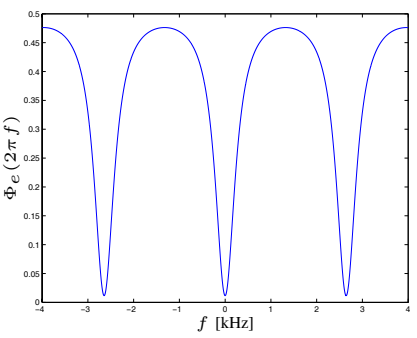

(a)

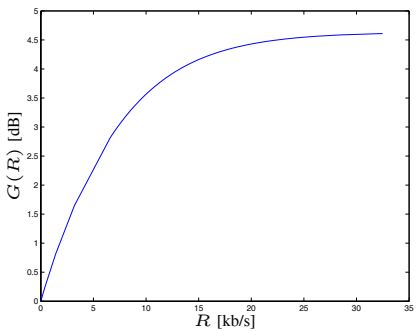

(b)
Fig. 3. Rate-constrained beamforming with an interfering source. (a) The power spectral density $\Phi_{e}$ used for reverse "water-filling". (b) The corresponding gain-rate function.

where we define

$$
\Delta(\Omega)=1-\cos \left(\Omega\left(\tilde{\tau}_{1,0}-\tau_{1,0}\right)\right)
$$

with

$$
\begin{gathered}
\tau_{1,0}=\tau_{1}-\tau_{0}=\frac{l \sin \alpha_{S}}{c} \\
\tilde{\tau}_{1,0}=\tilde{\tau}_{1}-\tilde{\tau}_{0}=\frac{l \sin \alpha_{I}}{c} .
\end{gathered}
$$

In this scenario, the optimal rate-allocation strategy obtained by reverse "water-filling" over $\Phi_{e}$ depends on both the direction of the source and the interferer and the distance between the hearing devices. Unlike Section IV-A, we observe that even if the involved PSDs are flat, the corresponding bitallocation is not uniform over the frequency band. For given directions $\alpha_{S}$ and $\alpha_{I}$, we first code the frequencies for which the array provides better rejection of the interfering signal. In this sense, the encoder contributes to the beamforming process by eliminating the least important frequency components such as to lower the communication bit-rate. This fact is illustrated in Figure 3(a) using the signal and noises defined by Equations (17) and (18), $\Phi_{I}$ given by

$$
\Phi_{I}(\Omega)=\sigma_{I}^{2} 1_{\left[-\Omega_{0}, \Omega_{0}\right]}(\Omega)
$$

and the following parameters: $l=0.2[\mathrm{~m}], c=340[\mathrm{~m} / \mathrm{s}]$, $\alpha_{S}=0$ [deg], $\alpha_{I}=40$ [deg], $f_{0}=\Omega_{0} /(2 \pi)=4000$ [Hz], $\sigma_{S}^{2}=\sigma_{I}^{2}=1$ and $\sigma_{N}^{2}=0.1$. The minima are located at the frequencies $\Omega$ verifying $\Delta(\Omega)=0$, i.e. for which $S$ and $I$ cannot be disambiguated. The corresponding gainrate function is plotted in Figure 3(b). Keeping the above parameters, we plot in Figure 4(a) the gain-rate function obtained for different values of $\alpha_{I}$ to illustrate the effect of an interferer on the reconstructed signal. In the vicinity of $S$, the beamforming gain decreases as $\alpha_{I}$ tends to $\alpha_{S}$ since it becomes difficult to spatially separate the desired signal from the interferer. We also show in Figure 4(b) the effect of the array's spatial extent $l$ on the gain-rate function. Increasing the distance between the microphones provides better directivity hence larger gains. For comparison, we provide the gain achieved with $l=0.02[\mathrm{~m}]$, the typical maximum possible distance allowing the two microphones to be embedded on the same hearing aid. We observe the significant gains that 


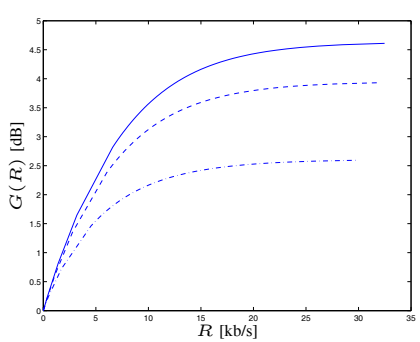

(a)

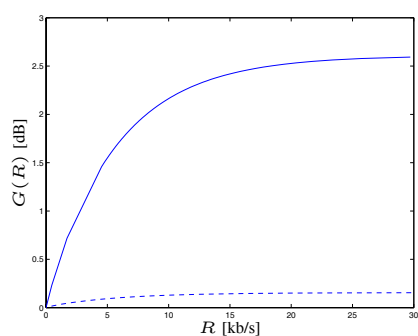

(b)
Fig. 4. Gain-rate with an interfering source. (a) For $\alpha_{S}=0$ [deg] and $\alpha_{I}=10$ [deg] (plain), $\alpha_{I}=8$ [deg] (dashed) and $\alpha_{I}=5$ [deg] (dashdotted). (b) For $\alpha_{S}=0$ [deg] and $\alpha_{I}=10$ [deg] with $l=0.2[\mathrm{~m}]$ (plain) and $l=0.02[\mathrm{~m}]$ (dashed).

can be achieved by exploiting the spatial extent offered by a wireless communication link between two hearing aids.

\section{CONCLUSION}

In this paper, we have investigated the beamforming gain provided by hearing aids that are allowed to collaborate using a wireless link. The problem has been identified and solved from an information-theoretic viewpoint. The impact of limited communication bit-rates on the beamforming capability of the hearing aids setup has been explored. Current research is focusing on extending the analysis provided in this paper to more general arrays.

\section{ACKNOWLEDGMENT}

Discussions with E. Telatar are gratefully acknowledged. This research was supported by the National Competence Center in Research on Mobile Information and Communication Systems (NCCR-MICS, http://www.mics.org), a center supported by the Swiss National Science Foundation under grant number 5005-67322.

\section{APPENDIX}

The proof of Theorem 1 makes use of the following lemma which can be easily demonstrated:

Lemma 1: Under the Gaussian assumptions made in this paper, we have

$$
R_{S \mid X_{0}}-R_{S \mid X_{0}, X_{1}}=R_{S}-R_{S \mid X_{1}^{e}}
$$

where $X_{1}^{e}=X_{1}-\mathrm{E}\left[X_{1} \mid X_{0}\right]$.

Proof of Theorem 1: It was shown in [9] that an optimal solution must be such that the MMSE is given by

$$
\mathrm{E}\left[\|S-\hat{S}\|^{2}\right]=\operatorname{tr}\left(R_{S \mid X_{0}}\right)-\sum_{i=m_{1}-k_{1}+1}^{m_{1}} \tilde{\lambda}_{i}
$$

where $\tilde{\lambda}_{i}$ denote the eigenvalues of the matrix $R_{S X_{1}^{e}}^{*} R_{S X_{1}^{e}} R_{X_{1}^{e}}^{-1}$ arranged in increasing order. Using Lemma 1 , we have that

$$
R_{S \mid X_{0}}-R_{S \mid X_{0}, X_{1}}=R_{S}-R_{S \mid X_{1}^{e}}=R_{S X_{1}^{e}} R_{X_{1}^{e}}^{-1} R_{S X_{1}^{e}}^{*} .
$$

Using the determinant formula $\operatorname{det}(A B+I)=\operatorname{det}(B A+I)$, it follows that the $m_{1}$ largest eigenvalues of $R_{S \mid X_{0}}-R_{S \mid X_{0}, X_{1}}$ are given by $\lambda_{i}=\tilde{\lambda}_{i}$, the $n-m_{1}$ remaining ones being zero. The MSE incurred by an optimal transform can thus be written as

$$
\mathrm{E}\left[\|S-\hat{S}\|^{2}\right]=\operatorname{tr}\left(R_{S \mid X_{0}, X_{1}}\right)+\sum_{i=1}^{m_{1}-k_{1}} \lambda_{i} .
$$

It remains to show that the transform given by (3) provides this MSE. We can write

$$
\begin{aligned}
& \mathrm{E}\left[\|S-\hat{S}\|^{2}\right] \\
&=\mathrm{E}\left[\left\|S-\mathrm{E}\left[S \mid K_{1} X_{1}, X_{0}\right]\right\|^{2}\right] \\
&=\mathrm{E}\left[\left\|S-\mathrm{E}\left[S \mid X_{0}\right]-\mathrm{E}\left[S \mid K_{1} X_{1}^{e}\right]\right\|^{2}\right] \\
&=\quad \mathrm{E}\left[\| S-R_{S X_{0}} R_{X_{0}}^{-1} X_{0}\right. \\
&\left.-R_{S X_{1}^{e}} K_{1}^{*}\left(K_{1} R_{X_{1}^{e}} K_{1}^{*}\right)^{-1} K_{1} X_{1}^{e} \|^{2}\right] \\
&= \operatorname{tr}\left(R_{S \mid X_{0}}\right) \\
&-\operatorname{tr}\left(R_{S X_{1}^{e}} K_{1}^{*}\left(K_{1} R_{X_{1}^{e}} K_{1}^{*}\right)^{-1} K_{1} R_{S X_{1}^{e}}^{*}\right) \\
&= \operatorname{tr}\left(R_{S \mid X_{0}}\right)-\sum_{i=m_{1}-k_{1}+1}^{m_{1}} \lambda_{i} \\
&= \operatorname{tr}\left(R_{S \mid X_{0}, X_{1}}\right)+\sum_{i=1}^{m_{1}-k_{1}} \lambda_{i}
\end{aligned}
$$

where (34) and (35) follow from properties of the conditional expectation in the jointly Gaussian case. Equation (36) follows from the definition of the Frobenius norm, the fact that expectation and trace commute and from the orthogonality of $X_{0}$ and $X_{1}^{e}$ (orthogonality principle). Finally, Equation (37) follows from the properties of the trace and the definition of $\bar{K}_{1}$. Since $\mathrm{E}\left[S \mid K_{1} X_{1}, X_{0}\right]=\mathrm{E}\left[S \mid K_{1} X_{1}^{e}, X_{0}\right]$, the availability of $X_{0}$ at the encoder does not change the MMSE. In this case, the optimal transform is still given by (3) but the transmitted coefficients are different since the rcKLT can be applied on $X_{1}^{e}$ instead of $X_{1}$.

\section{REFERENCES}

[1] H. L. V. Trees, Optimum Array Processing. Part IV of Detection, Estimation, and Modulation Theory. Wiley, New-York, 2002.

[2] D. H. Johnson and D. E. Dudgeon, Array Signal Processing : Concepts and Techniques. Prentice Hall, New Jersey, 1993.

[3] V. Hamacher, J. Chalupper, J. Eggers, E. Fischer, U. Kornagel, H. Puder, and U. Rass, "Signal processing in high-end hearing aids: State of the art, challenges, and future trends," EURASIP Journal on Applied Signal Processing, vol. 18, pp. 2915-2929, 2005.

[4] T. Flynn and R. Gray, "Encoding of correlated observations," IEEE Trans. Inform. Theory, vol. 33, no. 6, pp. 773-787, November 1987.

[5] H. Yamamoto and K. Itoh, "Source coding theory for multiterminal communication systems with a remote source," Trans. IECE Japan, vol. E63, no. 10, pp. 700-706, October 1980.

[6] S. C. Draper, "Successive structuring of source coding algorithms for data fusion, buffering and distribution in networks," Ph.D. dissertation, Massachusetts Institute of Technology, June 2002.

[7] D. Rebollo-Monedero, S. Rane, and B. Girod, "Wyner-Ziv quantization and transform coding of noisy sources at high rates," in Asilomar Conference on Signals, Systems, and Computers, November 2004.

[8] M. Gastpar, P.-L. Dragotti, and M. Vetterli, "The distributed, partial, and conditional Karhunen-Loève transforms," in IEEE Data Compression Conference, March 2003.

[9] K. Zhang, "Best linear unbiased estimation fusion with constraints," Ph.D. dissertation, University of New Orleans, December 2003.

[10] T. Berger, Rate Distortion Theory: A Mathematical Basis for Data Compression. Englewood Cliffs, NJ:Prentice-Hall, 1971. 